\title{
DEVELOPMENT OF COMPACT EUV SOURCE BASED ON LASER COMPTON SCATTERING*
}

\author{
S. Kashiwagi\#, R. Kato, J. Yang, G. Isoyama, \\ ISIR, Osaka University, Ibaraki, 8-1 Osaka 567-0047, Japan \\ K. Sakaue, A. Masuda, T. Nomoto, T. Gowa, M. Washio, \\ RISE, Waseda University, 3-4-1 Okubo, Shinjuku, Tokyo 169-8555, Japan \\ R. Kuroda, AIST, 1-1-1 Umezono, Tsukuba, Ibaraki 305-8568, Japan \\ J. Urakawa, KEK, 1-1 Oho, Tsukuba, Ibaraki 305-0801, Japan
}

\begin{abstract}
High-power extreme ultra-violet (EUV) sources are required for next generation semiconductor lithography. We start developing a compact EUV source in the spectral range of 13-14 nm, which is based on laser Compton scattering between a $7 \mathrm{MeV}$ electron beam and a high intensity $\mathrm{CO}_{2}$ laser pulse. Before the main laser Compton scattering for EUV radiation, the electron beam is prebunched by a high power seeding laser pulse with the Compton wavelength at a harmonic of the seeding laser [1]. In this paper, we describe the preliminary consideration for the EUV source development and a plan of experiment generating micro-bunched electron beam.
\end{abstract}

\section{INTRODUCTION}

The extreme ultra-violet (EUV) lithography that used a light source of wavelength $13.5 \mathrm{~nm}$ is the strongest candidate of the next generation lithography for LSI processing. The developments of EUV light sources using both laser produced plasma (LPP) and discharge produced plasma (DPP) are currently under way in the world [2]. Using the LPP or the DPP, the EUV light is obtained from high-temperature and high-density plasma. In these EUV sources, the debris problem is most critical issue and it results in reduced lifetime of expensive components and collector mirrors.

As an EUV source based on electron beam, a selfamplified spontaneous emission (SASE) free electron laser (FEL) using superconducting linear accelerators also had been demonstrated in the DUV and EUV spectral range with GW power (@ 32nm) at DESY, Hamburg [3]. The SASE FEL concept eliminates the need for an optical cavity, therefore there are no apparent limitations, which would obstruct operating at short wavelength range and increasing the average output power of this source. However, the SASE FEL scheme requires a large scale ( $\mathrm{GeV}$ order scale) accelerator and a very long undulator.

On the other hand, one of the promising approaches to intense and short-pulse EUV generation in the spectral range 13-14 nm is the use of a laser synchrotron source (LSS), which is based on laser Compton scattering. Laser Compton scattering between an electron beam and a highintense laser beam has been investigated as an effective

\footnotetext{
* This work of the supported by MECSST "High Tech Research Center" project No.707

\# shigeruk@sanken.osaka-u.ac.jp
}

technique for high-brightness short wavelength light generation [4-7].

As other proposal of EUV source, Intel Corp. (M. Goldstein et al.) had proposed a scheme of EUV source using a hybrid optical klystron for micro-bunching and high-gain harmonic generation FEL with laser seeding [1]. This scheme is a combination of pre-bunching and coherent radiation. As other scheme to generate shortwavelength radiation with small equipments, Bonifacio et al. have performed theoretical and numerical studies about quantum effect in SASE FEL with laser wiggler and the experimental parameters were specified for both the quantum and the classical regimes [8].

In this paper, we describe the preliminary studies about laser Compton scattering with coherent effect using prebunched beam and an experimental plan will be briefly introduced.

\section{LASER COMPTON SCATTERING FOR EUV GENERATION}

\section{Classical laser Compton scattering}

A low energy electron beam counter-propagates with respect to a high power laser pulse which produces an electromagnetic wave and the electron beam back-scatters the laser photons, with a frequency upshifted by a factor of $4 \gamma^{2}$ where $\gamma$ is a Lorentz factor. The LSS possesses several advantages, a compactness of instruments, wide energy tunability, a good directivity of the beam and a narrow spectrum of the EUV light, which can be obtained by selecting the scattered angles. Figure 1 shows the relation of the scattered angle and wavelength of EUV light via laser Compton scattering.

The total number of photons produced by the laser Compton scattering can be estimated using the product of the cross section of Compton scattering $\left(\sigma_{\text {Comp }}\right)$ and luminosity (L), which are determined by the scattering geometry of the electron beam and laser pulse. It is not possible to use this laser Compton configuration for a high-power EUV source directly because of the small cross section of Compton scattering and a small number of photons generated by one collision. We considered to applying a combination of a laser-stacking cavity using $\mathrm{CO}_{2}$ laser pulse and a low energy ERL (energy recovery linac) for EUV source. In numerical estimation, the photon flux of $10^{14}$ [photons $/ 2 \% \mathrm{~B}$.W./sec] in the EUV spectral range can be achieved using this scheme [9]. 


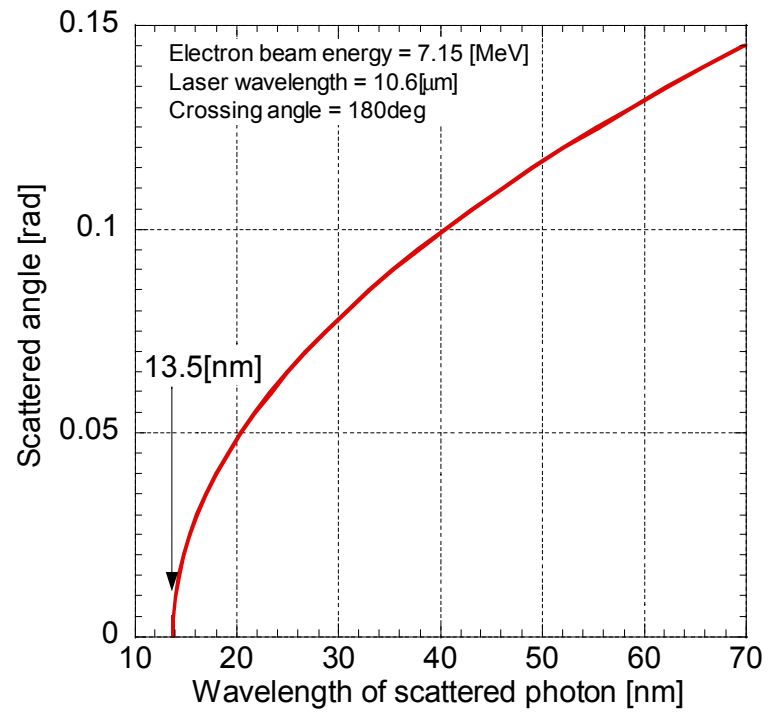

Fig. 1: Scattered angle and wavelength via inverse Compton scattering between electron beam and $\mathrm{CO}_{2}$ laser.

Huang and Ruth also proposed a compact laser-electron storage ring for x-ray generation in 1997 [10].

\section{Optical undulator}

In laser Compton configuration, an optical undulator is used instead of a magnetic undulator used in the usual configuration of the SASE experiments. For an optical undulator, the radiation wavelength $\lambda_{\mathrm{r}}$ is

$$
\lambda_{r}=\frac{\lambda_{L} \cdot\left(1+a_{0}^{2}\right)}{4 \gamma^{2}}
$$

where $\mathrm{a}_{0}$ is a normalized vector potential, which can be written by $a_{0}=0.85 \times \lambda_{L}[\mu \mathrm{m}] \sqrt{I}\left[\mathrm{~W} / \mathrm{cm}^{2}\right]$ (I: laser intensity $\left(I=P /\left(\pi w_{0}{ }^{2}\right)\right.$, P: laser output power, $\mathrm{w}_{0}$ : beam radius at beam waist). The wavelength $\lambda_{\mathrm{r}}$ differs from the resonance wavelength of conventional FEL by a factor two, since the magnetic undulator period $\lambda_{\mathrm{W}}$ is replaced by $\lambda_{\mathrm{L}} / 2$. We apply a high-power $\mathrm{CO}_{2}$ laser for laser Compton scattering to generate EUV light and the resonance energy of electron beam is approximately $7 \mathrm{MeV}$. In the optical undulator, the number of photons which one electron emits by an undulator radiation is given by

$$
N_{p h}=\frac{2}{3} \pi \alpha N_{W} K^{2}\left(1+K^{2}\right)
$$

where $\alpha, \mathrm{N}_{\mathrm{W}}$, and $\mathrm{K}$ are the fine structure constant (1/137), the number of undulator periods and undulator parameter $\left(=\mathrm{a}_{0}\right)$, respectively [11]. Assuming that $\mathrm{K}=0.5$ and $\mathrm{N}_{\mathrm{W}}=$ 100 , one electron approximately radiates 0.5 photons. In order to realize an EUV light source of the $100 \mathrm{~W}$ output, which corresponds to the EUV flux of $7 \times 10^{18}$ photons/sec, it is estimated that an average beam current of 2 amperes is required. This thing suggests it is difficult to generate enough number of photon for the high power EUV source using the conventional undulator radiation.

\section{SASE-FEL process using optical undulator}

The essential feature of the high-gain FEL including SASE is that a large number of electrons radiate coherently. In that case, the intensity of the radiation field grows quadratically with the number of electrons: $I_{n}=$ $n^{2} I_{1}$.

A possibility of high-gain FEL using the optical undulator examined as following. FEL parameter $(\rho)$ in one-dimensional model of SASE-FEL is calculated from the parameters of electron beam and laser pulse shown in Table 1. The number of optical undulator periods $\left(\mathrm{N}_{\mathrm{W}}\right)$ to reach the high-gain regime is in the order of $1 / \rho$ and the required length of the optical undulator is $\mathrm{L}_{\mathrm{W}}=\lambda_{\mathrm{L}} / \rho$. In the case of radiation wavelength at $13.5 \mathrm{~nm}, \rho \sim 9 \times 10^{-6}$ and $\mathrm{L}_{\mathrm{W}} \sim 0.6 \mathrm{~m}$, then $\mathrm{a}_{0} \sim 0.72$ with $500 \mathrm{GW}$ of the laser power. The FEL parameter is quite small and $\mathrm{L}_{\mathrm{w}}$ is much longer than the Rayleigh range (3mm) of the $\mathrm{CO}_{2}$ laser. In addition, it is necessary to satisfy the following conditions in 1D SASE-FEL model. There are (a) $\varepsilon<\lambda / 4 \pi$, (b) $\lambda \mathrm{L}_{\mathrm{g}} / 4 \pi \beta<\varepsilon$ (the gain length smaller than Rayleigh of the radiation), (c) $\sigma_{\gamma} / \gamma<\rho$, and the condition of the emittance is extremely severe. The condition (a) requires $10^{-8}$ order of the normalized emittance. A more realistic parameter search will be necessary to apply the high-gain FEL process to laser Compton scheme directly.

\section{Laser Compton scattering using pre-bunched beam}

In SASE process, the train of micro-bunches is ultimately formed with a bunch-spacing equal to the wavelength of radiation. The particles within a microbunch radiate coherently. We apply a pre-bunched beam for laser Compton scattering in order to enhance the radiation power and to shorten the optical undulator length.

In the case of EUV light source, it is necessary to form the micro-bunch with bunch spacing of $13.5 \mathrm{~nm}$ or its harmonic. A high power laser will be an effective beam density modulation source for the low energy electron beam in order to realize the micro-bunch. The initial energy spread of electron bunch near a cathode (thermal energy) is should be smaller than the modulation energy by the laser pulse otherwise there is no effect of the laser

Table 1: Parameters of electron beam and $\mathrm{CO}_{2}$ laser

\begin{tabular}{|c|l|}
\hline Electron beam & \\
\hline Beam energy & $7.2 \mathrm{MeV}$ \\
\hline Peak current & $1 \mathrm{kA}$ \\
\hline Beam size & $50 \mu \mathrm{m}$ \\
\hline $\mathrm{CO}_{2}$ laser & \\
\hline Wavelength & $10.6 \mu \mathrm{m}$ \\
\hline Peak power & $500 \mathrm{GW}$ \\
\hline Beam size & $50 \mu \mathrm{m}$ \\
\hline
\end{tabular}

U04 Other Applications 

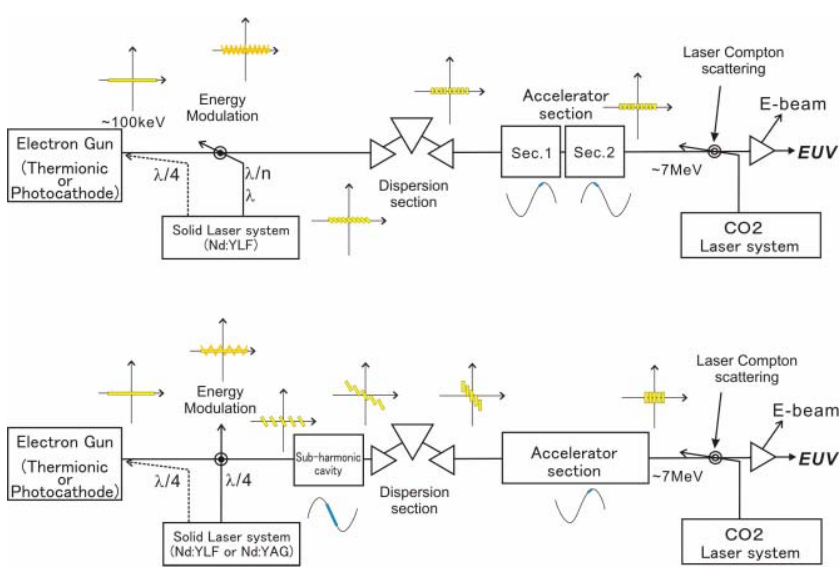

Fig.2: Schematic drawing of laser Compton scattering with pre-bunched electron beam. (The upper scheme based on the Intel FEL [1])

bunching. In Refs. 1 and 12, the intensity modulation using a laser field is described. On the other hand, we will try to estimate an effect of pre-bunching and how much density modulation of electron beam is necessary to enhance the EUV radiation power using Genesis 1.3.

\section{EXPERIMENT OF ELECTRON PRE- BUNCHING USING HIGH-POWER LASER}

Now, two schemes are considered to be a candidate for the EUV source based on laser Compton scattering as shown in Fig. 2. One is based on Intel FEL scheme, in which non-relativistic electron beam is bunched with a harmonics period of the optical undulator using an optical klystron, and EUV light is generated by a high-gain harmonic generation. The spacing of the micro-bunch is fixed at the optical klystron, and the micro-bunches are formed by a velocity bunching and a magnetic compressor. In another scheme, an electron beam and a focused laser interact perpendicularly with each other and the electron beam energy is modulated by an electric field of the laser [12]. At a drift space of downstream, the energy modulation converts to density modulation by the velocity bunching, furthermore the bunch-spacing are compressed by an rf slope of a sub-harmonic cavity and a magnetic chicane. In this scheme, final bunching spacing is determined the rf amplitude of the sub-harmonic cavity and the transfer matrix in longitudinal direction of the chicane. The electron beam is accelerated to $7 \mathrm{MeV}$ without a growth of energy spread in micro-bunch, therefore an accelerating phase of main structure are precisely adjusted in the both schemes. The condition of the energy spread for electron beam at the optical undulator is severe and its requirement is less than $10^{-5}(\rho)$ which come from the condition (c) in the previous section. We will perform an experiment of electron pre-bunching using an electron gun and a solid laser system. Firstly Intel scheme of micro-bunching will be studied. The electron beam with 10 ps (FWHM) is generated using a
$100 \mathrm{kV}$ DC photo-cathode electron gun with 4th harmonics of a Nd:YLF laser, and the Nd:YLF laser is used as a seeding laser for the energy modulation. An influence of a space charge on the micro-bunching will be studied in detail.

\section{SUMMARY}

We just start developing the EUV source based on laser Compton scattering. In the preliminary consideration, there were very severe condition for the average current and the emittance of electron beam. In order to enhance the intensity of radiation to use coherent effect, the prebunched beam is applied to the laser Compton scheme. The EUV light is radiated by the micro-bunch coherently. The experiment of micro-bunching will be tested using the DC photocathode gun and high-power Nd:YLF laser. The numerical study is also performed about the microbunching effect on the laser Compton scattering and the space charge effect in micro-bunching.

\section{ACKNOWLEDGMENTS}

The authors would like to express sincere thanks to Dr. A. Endo of GIGAPHOTON Inc. for his great help and suggestions for the EUV source development. This work of the supported by MECSST "High Tech Research Center" project No.707.

\section{REFERENCES}

[1] M. Goldstein et al., Proc. of the 27th Int. FEL conference, Stanford, California, USA (2005) pp.422-425.

[2] A. Endo, SEMATECH EUV Source Workshop, Barcelona, Spain (2006)

[3] V. Ayvazyan et al., Eur. Phys. J. D 37 (2006) 297-303.

[4] W. P. Leemans, R. W. Schoenlein, P. Volfbeyn, A. H. Chin, T. E. Glover, P. Balling, M. Zolotorev, K. J. Kim, S. Chattopadhyay, and C. V. Shank, Phys. Rev. Lett. 77, 4182 (1996).

[5] A. Ting, R. Fischer, A. Fisher, K. Evans, R. Burris, J. Krall, E. Esarey, and P. Sprangle, J. Appl. Phys. 78, 575 (1995).

[6] S. Kashiwagi et al., J. Appl. Phys. 98 (12), (2006) 123302

[7] S. Kashiwagi et al., Nucl. Instrum. Methods A 455 (2000) 36.

[8] R. Bonifacio et al., Phys. Rev. ST-AB 9, 090701 (2006).

[9] K. Sakaue et al., SEMATECH EUV Source Workshop, Barcelona, Spain (2006)

[10] Zh. Huang, R. D. Ruth, Phys. Rev. Lett., 80 (1998) 976.

[11] X-ray data booklet, Lawrence Berkeley Nat. Lab.

[12] T. Shintake, Proc. of FEL 2006, BESSY, Berlin, Germany (2006) pp.242-247. 